\title{
Experimental Determinations of Coherent Multidimensional Vibrational Spectroscopy
}

\author{
Daniel Besemann, Nicholas Condon, Kent Meyer, Wei Zhao, and John C. Wright ${ }^{\star}$ \\ Department of Chemistry, Chiversity of Wisconsin-Madison. Madison. W7 53709. LSA \\ Received.April 14, 2003
}

\begin{abstract}
Coherent multidimensional vibrational spectroscopy is a new technique for establishing correlations between features in vibrational spectra that are caused by intra- and intermolecular interactions. These interactions cause cross-peaks between vibrational transitions that reflect the coupling. In this paper. we use Doubly Vibrationally Enhanced Infrared Spectroscopy (DOVE-R) and DOVE-Raman processes to obtain coherent two dimensional vibrational spectra. The spectra are fitted to obtain the dephasing rates and third order susceptibilities $\left(\chi^{3}\right)$ for the nonlinear processes. We show that the DOVE $\chi^{(3)}$ values are directly related to the molar absorptivities and Raman $\chi^{i 3\rangle}$. We then use these relationships to obtain estimates for the $\chi^{\prime 3 \prime}$ of the stimulated photon echo and $\chi^{\prime \prime \prime}$ of the six wave mixing spectroscopies. respectively. We also predict the ratio of the cascaded four wave mixing signal to the six wave mixing signal.
\end{abstract}

Key Words : Nonlinear, Vibrational. Two dimensional, Coherent. Infrared

\section{Introduction}

Linear spectroscopy is based on establishing molecular resonances at specific frequencies which enhance the linear response of the molecular polarization that is induced by the electric field of an exciting light source. Nonlinear spectroscopy is based on perturbing the linear response with electric fields that are strong enough to change the linear optical properties of the molecule. The result is that the linear response is modulated at the frequency of the perturbing strong field so the induced polarization acquires new frequencies at the sum or difference of the original and the perturbing frequencies. The oscillating polarization that produces these new frequencies is enhanced when it is resonant with molecular states, either with states that are resonant with the first excitation or the second perturbing excitation. Two dimensional nonlinear spectroscopy is based on coupling between the two resonant states. Spectral features in two dimensional vibrational spectroscopy can only appear if there are intra- or intermolecular interactions that couple two vibrational modes. Otherwise it is not possible for one mode to modulate the optical properties associated with the other mode. When the two modes are coupled. cross-peaks appear in the two dimensional spectra at the frequency of each mode. It is this sensitivity to the presence of coupling interactions and the correlations they introduce that leads to the interest in two dimensional vibrational spectroscopy. 1.s The only features that one observes are those that are associated with interactions. One can eliminate the spectral congestion from all the other modes that may be much stronger in a normal one dimensional vibrational spectrum. ${ }^{\hat{3}}$

\section{Theory}

Quantum mechanically. the oscillating polarization of a linear spectroscopy corresponds to a coherence. a linear combination of the two states involved in the transition induced by the electromagnetic field. ${ }^{+6}$

$$
\Psi(x, t)=c_{a}(t) \Psi_{a}(t) e^{i(\omega k r}+c_{3}(t) \Psi_{3}(t) e^{i(a b r}
$$

Before the transition. the system is a population in state $a$ or $b$ and is described by the diagonal density matrix element. $\rho_{a u}$ or $\rho_{b b}|\Psi|^{2}$ is then time independent with no oscillatory component. In a transition, the electromagnetic field perturbs the system by mixing the states so $|\Psi|^{2}$ now has cross-terms that oscillate at $\left(\omega_{b}-\omega_{k}\right)$. This mixed state is called a colerence and it is described by the off-diagonal density matrix element. $\rho_{b a}=c_{b} c_{a}{ }^{*}$. State $b$ in $\rho_{b a}$ is the ket and state a is the bra. If the coherence has a funite dipole moment, $\mu_{b a}$, its oscillation will reradiate light at $\left(\omega_{b}-\omega_{a}\right)$. The polarization is related to the coherence by

$$
P=N F \mu_{l a} \rho_{l a}
$$

where $N$ is the molecular concentration and $F$ is a local field correction factor. The polarization is commonly described phenomenologically by the relationship

$$
P=\chi^{(1)} E+\chi^{(2)} E+\chi^{(3)} E^{3}+\ldots . .
$$

The reradiated electric field is given by

$$
E=\frac{4 \pi \omega P}{n c}
$$

with an intensity

$$
I=\frac{c n}{8 \pi} E^{2}
$$

Nonlinear spectroscopy perturbs the coherence by changing its mixture of states. The time evolution of a coherence along a nonlinear pathway is conveniently described by Liouville diagrams that map the sequential perturbations of the coherences and the fields that induced them. ${ }^{4}$ The relationships between the fields and the coherences are best defined by wave mixing energy level diagrams (WMEL diagrams). ${ }^{?}$ Figure 1 shows both the WMEL and Liouville diagrams for 
(a)

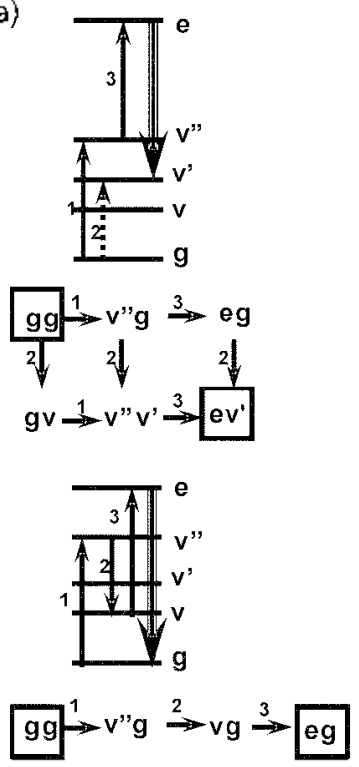

(b)

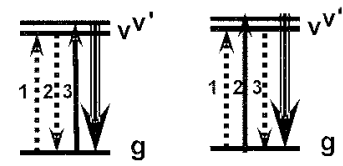

(c)

c) Six Wave Mixing
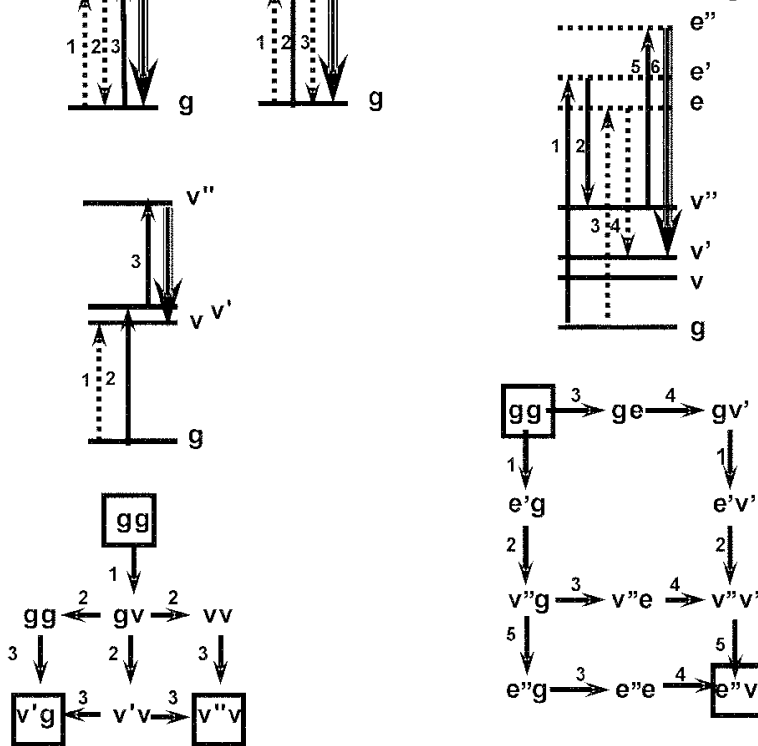

Cascaded Four Wave Mixing
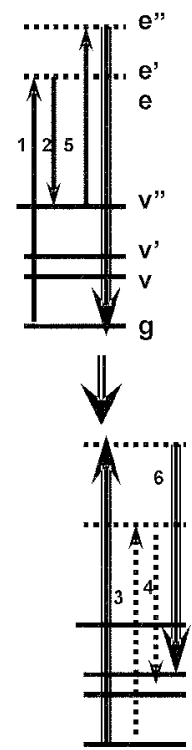

Figure 1. Wave mixing energy level (WMEL) and Liouville diagrams for doubly vibrationally enhanced four wave mixing (la), stimulated photon echo ( $\mathrm{lb}$ ), and six wave mixing ( lc) spectroscopies. The single letters indicate molecular states, the letter pairs indicate coherences or populations, the numbers designate excitation beams, the solid anows represent transitions on the ket side of a coherence while the dotted lines indicate bra side transitions. The triple arrows designate the output transition. The boses designate the initial population and the final emitting coherence.

the three multidimensional vibrational spectroscopies that are discussed in this paper. In this figure. the letters define the levels. the pair of letters defines the states involved in the coherence the numbers define the electromagnetic field. and the arrows show the transitions where states change. Note that it is possible to change either state (designated either the ket or bra states for the first or second states. respectively) in the coherence. Ket side transitions are denoted by solid lines and bra side transitions are denoted by dotted lines. Nonlinear spectroscopy is characterized by the number of interacting electromagnetic fields so two interactions mix two fields to create a second order polarization that radiates a third field and it is consequently called three wave mixing. For three wave mixing. symmetry considerations result in an equal number of opposing polarizations in an isotropic material that interfere destructively so no net radiated field occurs. ${ }^{8}$ The lowest order nonlinear process that can be observed in isotropic materials is four wave mixing where three fields interact to create a nonlinear polarization that radiates the fourth field.

The nonlinear excitations create an ensemble of molecular polarizations that have a phase relationship. The relationship can be defined by a wavelength and directionality that is linked to the excitation fields by momentum conservation. For a nonlinear process that creates an output frequency given by $\omega_{4}=\omega_{1}-\omega_{2}+\omega_{3}$, the wave-vector that describes the nonlinear polarization is given by $\bar{k}_{1}-\bar{k}_{2}+\bar{k}_{3}$ where $k$ $\equiv n \omega / c^{5}$. This relationship shows that the wavelength and directionality of the nonlinear polarization is defined by the wavelengths and directionality of the three excitation fields. The nonlinear polarization reradiates an electromagnetic field at $\omega_{4}$ with a wavelength that can be different from the polarization since the index of refraction at $\omega_{4}$ is different from the input fields. Thus the phase of the launched electromagnetic field can differ from the phase of the nonlinear polarization that launches it. Phase matching of the beams can still be achieved by appropriate choice of the directionality of the beans such that $\bar{k}_{4}=\bar{k}_{1}-\bar{k}_{2}+\bar{k}_{3}$.

\section{Dove Methods}

Our work in multiresonant four wave mixing spectroscopy has focused on using three different frequencies for the excitations so three separate transitions are possible. Triply resonant four wave mixing spectroscopy was implemented for systems where all the transitions involved electronic transitions. ${ }^{10.11}$ The methods had the selectivity and resolution characteristic of multidimensional nonlinear spectroscopies. ${ }^{11-15}$ However the involvement of electronic states required cryogenic temperatures in order to maintain sufficiently narrow transitions.

The work was extended to vibrational transitions because the smaller coupling to the bath allows vibrational states to remain sharp-lined at room temperature. ${ }^{16-22}$ Although there are many approaches for achieving coherent multidimensional vibrational spectroscopy (CMDVS), the strategy for creating the vibrational resonances in this work are diagrammed in Figure la. There are two processes that drive doubly vibrationally enhanced (DOVE) FWM- DOVE-IR and DOVERaman. ${ }^{21.22}$ In DOVE-IR (top diagrams in Figure la), two infrared excitations drive the ket and bra states to create a double quantum coherence. i.e. a linear combination of the two resonant vibrational states. A third excitation drives the Raman transition that creates the coherence which radiates 
the output field. There are three pathways from the initial population represented by $\rho_{\mathrm{gg}}$ to the output coherence. $\rho_{\mathrm{r}}$. that depend upon the time ordering of the three transitions. In DOVE-Raman (bottom diagrams in Figure la). the infrared excitations create two vibrational transitions on the ket state and the third excitation drives the output Raman transition. $\rho_{\text {eg }}$ also on the ket state. There is only one pathway to the output coherence. The phases from each of the DOVE-IR and DOVE-Raman pathway's define the amplitude level interference that occurs between the pathways. The amplitudes are proportional to the off-diagonal $\rho$ elements describing the output coherence.

As an example. the amplitude level enhancement for a DOVE-IR process is proportional to $\rho_{\omega}$. The expression for the steady state value of $\rho_{e r}$ that includes the two $\rho_{e v}$ doubly vibrationally resonant coherence pathway's is:

$\rho_{c t b}=$

$$
\begin{aligned}
& \frac{\mu_{g c} \mu_{c i d} \mu_{g b} \rho_{g g} E_{1} E_{2} E_{3}}{8 \pi^{3}} \sum_{\text {8.b.c.cil=sll states }}\left(-\frac{1}{\Delta_{c g} \Delta_{c b} \Delta_{d b}}+\frac{1}{\Delta_{b g}^{2} \Delta_{c b} \Delta_{c l b}}\right) \\
& \times \exp \left(i\left[\left(k_{1}-k_{2}+k_{3}\right) z-\left(\omega_{1}-\omega_{2}+\omega_{3}\right) t\right]\right)
\end{aligned}
$$

Here. $\Delta_{c g} \equiv \omega_{c g}-\omega_{1}-i \Gamma_{c g}, \Delta_{c b} \equiv \omega_{s}-\omega_{1}+\omega_{2}-i \Gamma_{c b} . \Delta_{c b} \equiv$ $\omega_{b b j}-\omega_{4}-i \Gamma_{b j}, \Delta_{b g}^{*} \equiv \omega_{b g}-\omega_{2}+i \Gamma_{b g}, \Delta_{a g} \equiv \omega_{q g}-\omega_{1}+\omega_{2}-$ $i \Gamma_{a g} . E$ is the electric field of the excitation sources. and $\mu_{j j}$. $\omega_{i j}$ and $\Gamma_{i, j}$ are the transition moment. transition frequency. and dephasing rate for the $i-j$ transition, respectively: The summation over all states in equation 6 simplifies for CMDVS methods because the resonant vibrational states dominate. For DOVE-IR we assume that state $c$ is a vibrational state $v^{\prime \prime}$ and state $b$ is a vibrational state $v$. For DOVE-Raman. we assume that state $c$ is a vibrational state $v^{\prime \prime}$ and state $b$ is a vibrational state $v$. For the examples in this paper. state $v^{\prime \prime}$ is a combination band of states $v$ and $v^{\prime}$ although many other choices are possible..$^{23}$ For both DOVEIR and DOVE-Raman. state $d$ is an effective electronic state. The doubly vibrationally resonant output polarization created by the $\rho_{d b}$ coherence is then given by

$$
\begin{aligned}
& \bar{r}=\frac{N F \mu_{v, e} \mu_{v,{ }_{g}} \mu_{v{ }^{\prime}{ }_{e}} \mu_{v, g} \rho_{g g} E_{1} E_{2} E_{3}}{8 \hbar^{2}} \\
& \times\left(\frac{1}{\Gamma_{v^{\prime \prime}{ }^{\prime} \Gamma_{v^{\prime \prime} v^{\prime}} \Delta_{v^{\prime \prime} \prime^{\prime}}}}+\frac{1}{\Gamma_{v^{\prime} g} \Gamma_{v^{\prime \prime} v^{\prime}} \Delta_{q^{\prime} \prime^{\prime}}}\right) \\
& \times \exp \left(i\left[\left(k_{1}-k_{2}+k_{3}\right) z-\left(\omega_{1}-\omega_{2}+\omega_{3}\right) t\right]\right) \text {. }
\end{aligned}
$$

The corresponding $\chi^{(3)}$ is

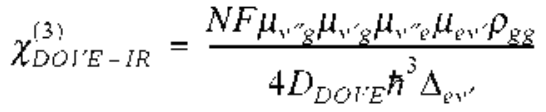

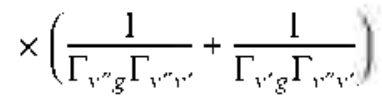

where $F$ is the local field factor. $N$ is the number density. and $D$ is a degeneracy factor that reflects the Maker-Terhune convention for $\chi^{1}$

Equation 7 predicts cross-peaks at frequencies that are resonant with two coupled vibrational states. As shown earlier. there are four transitions that occur in a FWM experiment and at least one of them involves a transition that is forbidden for harmonic systems. Consequently, if there is no coupling that can cause an anhannonicity. no cross-peak occurs. Thus. at least one of the transitions must involve a combination band. ${ }^{23}$ In our examples. the $\omega_{1}$ frequency is resonant with a combination band. $g \rightarrow v^{\prime \prime}$, which is a mixture of the $v$ and $v^{\prime}$ fundamentals. The $\omega_{2}$ excitation is resonant with the $g \rightarrow v^{\prime}$ fundamental. The $\omega_{4}-\omega_{2}$ frequency is resonant with the $\left(v^{\prime}+v \rightarrow v^{\prime}\right)$ Raman hot band transition. For the three transitions. the vibrational quantum number change. $\Delta v$ is 2,1 , and -1 for $\omega_{1} . \omega_{2}$, and $\omega_{3}-\omega_{4}$ induced transitions. respectively:

\section{Results}

Figure 2 shows the two dimensional DOVE-FWM spectra of a mixture of $\mathrm{CH}_{3} \mathrm{CN}$. $\mathrm{CD}_{3} \mathrm{CN}$. and $\mathrm{C}_{6} \mathrm{D}_{6}$ along with the infrared spectrum in the region of the DOVE resonances. ${ }^{2+}$ The $x$ and $y$ axes represent the $\omega_{1}$ and $\omega_{2}$ frequencies. respectively. and the colors indicate the signal intensity at $\omega_{4}$. Multiple color cycles are used so spectral changes are more visible. The two prominent peaks result from DOVEIR transitions with the $\mathrm{C} \equiv \mathrm{N}$ stretch fundamental (vis) and the $\mathrm{C} \equiv \mathrm{N}$ and $\mathrm{C}-\mathrm{C}\left(v_{4}\right)$ stretch combination band of $\mathrm{CH}_{3} \mathrm{CN}\left(\omega_{1}=\right.$ $3165 \mathrm{~cm}^{-1}:\left(\omega_{2}=2254 \mathrm{~cm}^{-1}\right)$ and $C_{3} \mathrm{CN}\left(\omega h=3090 \mathrm{~cm}^{-1}\right.$. $\left.\omega=2264 \mathrm{~cm}^{-1}\right)$. There are diagonal features extending through these features that come from the Raman process when $\omega_{1}-\omega_{2}$ matches the $\mathrm{C}-\mathrm{C}$ stretch vibrational modes at $828 \mathrm{~cm}^{-1}$ and $917 \mathrm{~cm}^{-1}$ for $\mathrm{CD}_{3} \mathrm{CN}$ and $\mathrm{CH}_{3} \mathrm{CN}$. respectively. When $\omega_{1}$ is far from vibrational resonances. one has nonmal colerent anti-Stokes Raman scattering (CARS) that involves a nonresonant intermediate electronic state and a Raman resonance with the $\mathrm{C}-\mathrm{C}$ stretch. However, when $\omega_{3}$ is near a vibrational resonance that is coupled to the $\mathrm{C}-\mathrm{C}$ stretch

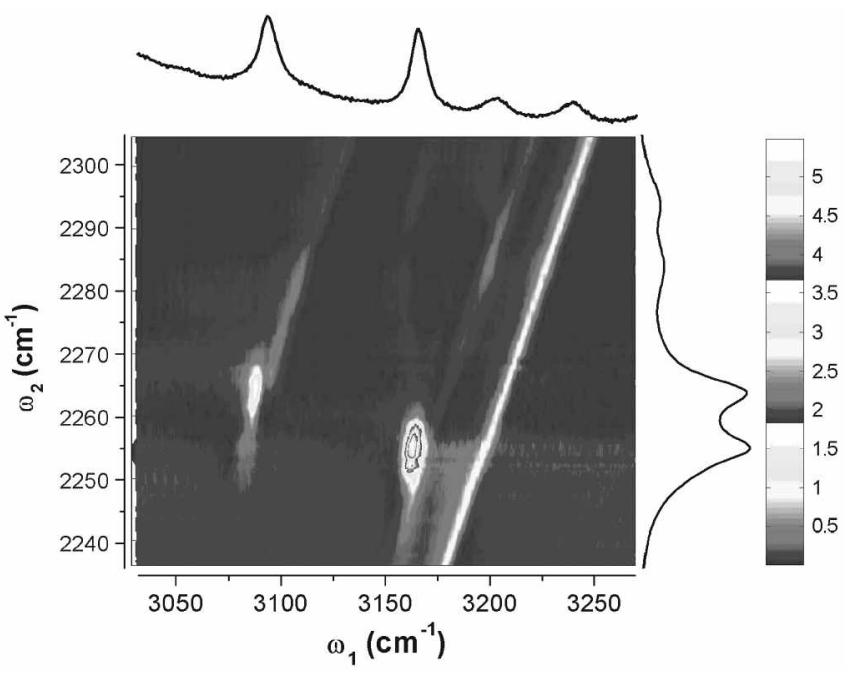

Figure 2. 2D-DOVE spectrum of a $\mathrm{CH}_{3} \mathrm{CN}, \mathrm{CD}_{3} \mathrm{CN}, \mathrm{C}_{6} \mathrm{D}_{6}$ misture along with the infrared spectrum of the misture. ${ }^{2+}$ 

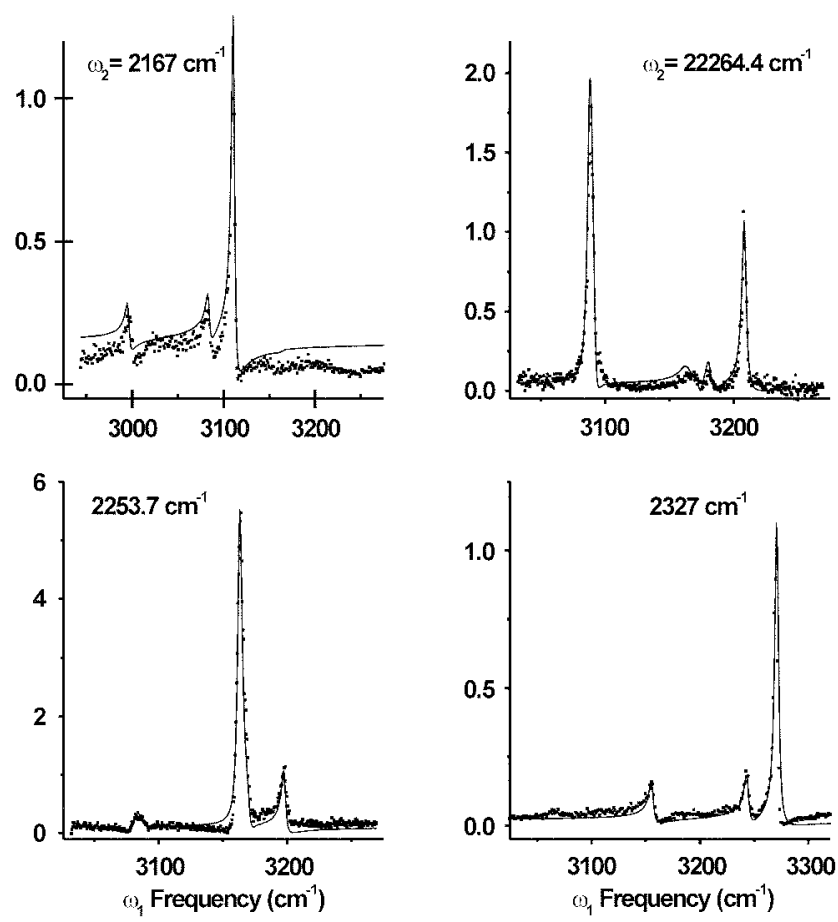

Figure 3. Examples of the experimental and theoretical spectra for the data in Figure 2. The spectra are plotted as a function of $\omega$, for particular values of $\omega_{n}{ }^{2}$

mode. there is a double enhancement from the DOVERaman process. These enhancements are seen when $\omega_{1}$ is 3165 or $3090 \mathrm{~cm}^{-1}$ for the $(C-C)+(C \equiv N)$ combination bands of $\mathrm{CH}_{3} \mathrm{CN}$ and $\mathrm{CD}_{3} \mathrm{CN}$. respectively and $3203 \mathrm{~cm}^{-1}$ for the $2 \times(\mathrm{C}-\mathrm{C})+\left(\mathrm{C}-\mathrm{H}\right.$ wag) $\left(v_{3}\right)$ combination band of $\mathrm{CH}_{3} \mathrm{CN}$. There is another diagonal ridge when $\omega_{3}-\omega_{2}$ matches the ring breathing mode of $\mathrm{C}_{6} \mathrm{D}_{6}$ at $944 \mathrm{~cm}^{-1}$. This feature is used as an internal standard for normalizing all of the spectra that together create Figure 2. Finally. there is a background over the entire spectnum that arises from the nonresonant electronic polarization.

There is amplitude level interference between all of the processes and this interference changes the line-shapes of the frequency dependent output intensity. Levenson and Bloembergen measured the third order nonlinear susceptibilities. $\chi^{3 j}$. of $\mathrm{C}_{6} \mathrm{H}_{6}$ by fitting the line-shape changes induced by the amplitude level interference. ${ }^{256}$ In a similar way. we have fit all the spectra in the two dimensional plot in Figure 2 with a common set of parameters in order to determine both the relative size of all the nonlinearities and the dephasing rates of the nonlinear coherences. ${ }^{37}$ Figure 3 shows examples of the experimental and theoretical lineshapes that result from this fitting. The first and last spectra are taken at $\omega_{2}$ frequencies that are far from vibrational resonances so the spectra do not contain any DOVE resonances. The middle two spectra were taken when $\omega \leq$ was resonant with either the $\mathrm{CH}_{3} \mathrm{CN}$ or $\mathrm{CD}_{3} \mathrm{CN} \mathrm{C} \equiv \mathrm{N}$ stretch modes. The deuterobenzene line at $944 \mathrm{~cm}^{-1}$ is alway's the line farthest to the right in the spectra and it alway's has the same intensity since there are no additional resonances that enhance it. Since $\chi^{(3)}$ of the benzene ring breathing mode is known. the $x^{(3)}$ values of all the DOVE processes can be determined. We find a value of a value of $3.4 \times 10^{-14} \mathrm{~cm}^{3 /} / \mathrm{erg}$ for the DOVE-IR process involving a double resonance with the C-C stretch mode and the C-C. C $\equiv \mathrm{N}$ combination band. Details of this procedure have been published elsewhere. ${ }^{27}$

\section{Relationships between Multidimensional and One Dimensional Methods}

It has also been shown that the CMDVS nonlinearities are directly related to the individual infrared. Raman. and hyperRaman transitions. ${ }^{1.22 .23}$ For example the DOVE-IR and DOVE-Raman processes can be described as two amplitude level infrared transitions and a single amplitude level Raman transition. The magnitude of the DOVE-IR $x^{3)}$ value is therefore directly related to the magnitude of the infrared absorption coefficients for the two infrared transitions and the magnitude of $\mathcal{X}^{(\mathrm{i})}$ for the Raman transition. In particular. the absorption coefficient is

$$
\alpha_{r^{\prime} g}=\frac{4 \pi \omega_{r^{\prime} g} N F_{r^{\prime} g} \mu_{r^{\prime} g}^{\prime} \rho_{g g}}{\hbar c \Gamma_{r^{\prime} g}}
$$

and the Raman $\chi^{\prime 3 !}$ is

$$
\chi_{\text {rg. Raman }}^{(3)}=\frac{-i N F_{e g}^{2} F_{e v 1} \mu_{e g}^{2} \mu_{e v}^{2} \rho_{g g}}{4 D_{\text {Raman }} \hbar^{3}\left|\Delta_{e g}\right|^{\hat{2}} \Gamma_{\gamma g}}
$$

where $\Delta_{g g}=\omega_{\mathrm{g} g}-\omega_{1}-i \Gamma_{i g,}, \omega_{1}=\omega_{3}$ and $\mathrm{c}$ is the speed of light. $D_{\text {Raman }}$ is 3 for a 2 laser experiment and 6 for a 3 laser experiment. Solving for the transition moments in eqns. 9 and 10 and substituting into the peak DOVE-IR $\chi^{\prime 3 !}$, one finds

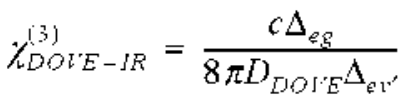

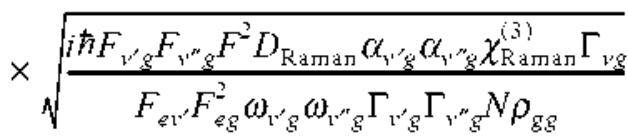

$$
\begin{aligned}
& \times\left(\frac{1}{\Gamma_{v^{\prime \prime} g} \Gamma_{v^{\prime \prime} v^{\prime}}}+\frac{1}{\Gamma_{v^{\prime} g} \Gamma_{r^{\prime \prime} v^{\prime}}}\right)
\end{aligned}
$$

Since the absorption coefficients and Raman susceptibilities are easily measured from normal vibrational spectra and are published elsewhere. it is easy to obtain reasonable estimates for $X_{D O S E-I R}^{(3)}{ }^{1.22 .23}$ For the dominant DOVE-IR peak in $\mathrm{CH}_{3} \mathrm{CN}$. we estimate a magnitude of $4.3 \times 10^{-14} \mathrm{~cm}^{3} / \mathrm{erg}$. This value is close to the experimental value of $3.4 \times 10^{-1+}$ $\mathrm{cm}^{3} / \mathrm{erg}$. More details on the procedures for obtaining these values are available elsewhere. ${ }^{77}$ In addition. Kwak. Cha. and Cho have calculated the $\chi_{D O V^{\prime} E-J R}$ for acetonitrile using two different $a b$ initio methods and determined the intramolecular coupling for all the modes. ${ }^{28}$ Their values of $7.7 \times$ $10^{-14}$ and $11.5 \times 10^{-1+} \mathrm{cm}^{3} / \mathrm{erg}$ agree with our measurement and our estimate. All of these approaches give confidence that we understand the mechanisms responsible for the $2 \mathrm{D}$ DOVE spectra. 


\section{Extensions to Stimulated Photon Echo}

This strategy for determining the CMDVS nonlinearities can be extended to all the different methods that are available for CMDVS and to the stimulated photon echo and six wave mixing or 2D Raman methods in particular. Hochstrasser and coworkers ${ }^{-9-38}$ and Tokmakoff and coworkers ${ }^{39-43}$ have developed time-domain heterody'ne-detected stimulated photon echo (SPE) methods that are triply resonant. Figure lb shows the Liousille and WMEL diagrams for SPE methods. There are several key ideas to understanding the SPE method. First it is fully resonant and each of the transitions is allowed since $\Delta y= \pm 1$ for all transitions. Second. there are multiple coherence pathways. some of which involve the fundamentals and others that involve the combination bands and/or overtones. These pathways destnuctively' interfere and can eliminate the cross-peaks if there is no coupling between the modes that are involved ${ }^{37.41}$ For example. the $g g \rightarrow g v \rightarrow g g \rightarrow v^{\prime} g$ pathway destructively interferes with the $g g \rightarrow g v \rightarrow v^{\prime} v \rightarrow v^{\prime \prime} v$ pathway and the two can cancel if their coherences' frequencies. dephasing rates. and transition probabilities are equal. Coupling results in frequency shifts or changes in the dy'namics so the pathway's become inequivalent a residual contribution remains. and a cross-peak appears. This mechanism for creating cross-peaks represents a different manifestation of coupling from DOVE methods where the combination band was involved. However. there must still be a relationship between the SPE and the DOVE processes because a coupling between modes will cause the combination band required for the DOVE process and an anharmonic shift that is required for the SPE process.

The $\chi^{(3)}$ expression for the SPE pathways in Figure $1 b$ is

$$
\begin{aligned}
& \chi_{S P E}^{(3)}=\frac{N F \rho_{g g}}{4 D_{S P E} h^{3} \Delta_{g}}
\end{aligned}
$$

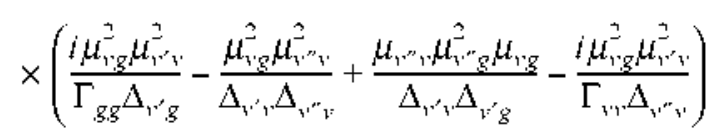

We evaluate $\chi^{33}$ using the parameters determined for acetonitrile's molar absorptivities and equation 9 to determine the

\section{Table 1 .}

\begin{tabular}{lcccc}
\hline States & $\begin{array}{c}\text { Dephasing } \\
\text { Rate } \\
\left(\mathrm{cm}^{-1}\right)\end{array}$ & $\begin{array}{c}\text { Frequency } \\
\left(\mathrm{cm}^{-1}\right)\end{array}$ & $\begin{array}{c}\text { Molar } \\
\text { absolptivity } \\
\left(\text { liter } \mathrm{M}^{-1} \mathrm{~cm}^{-1}\right)\end{array}$ & $\begin{array}{c}\text { Raman } \chi^{i 3)} \\
\left(\mathrm{cm}^{3} / \mathrm{erg}\right)\end{array}$ \\
\hline$v g\left(v=v_{4}\right)$ & 2.5 & 917 & 9.15 & $3.1 \times 10^{-15}$ \\
$v^{\prime} g\left(v^{\prime}=v_{2}\right)$ & 3.9 & 2254 & 41.7 & $8.5 \times 10^{-15}$ \\
$v^{\prime \prime} g\left(v^{\prime \prime}=v_{2}+v_{4}\right)$ & 4.7 & 3165 & 2.52 & - \\
$g g$ & $2.0^{\alpha}$ & 0 & - & - \\
$v^{\prime \prime} v^{\prime}\left(v_{2}+v_{4}, v_{2}\right)$ & 2.5 & 911 & 9.15 & $3.1 \times 10^{-15}$ \\
$v^{\prime \prime} v\left(v_{2}+v_{4}, v_{4}\right)$ & 3.9 & 2248 & 41.7 & $8.5 \times 10^{-15}$ \\
$v^{\prime} v\left(v_{2}, v_{4}\right)$ & 2.5 & 1337 & - & - \\
$v^{\prime} g\left(v^{\prime}=v_{3}\right)$ & 4.9 & 1376 & 20.2 & $4.7 \times 10^{-16}$ \\
$v^{\prime \prime} g\left(\mathrm{v}^{\prime \prime}=v_{3}+v_{4}\right)$ & 6.4 & 293 & 7.8 & $4 \times 10^{-16}$ \\
\hline
\end{tabular}

"Excitation laser bandwidth. transition moments required in equation 12. If the $g \rightarrow v$ and the $g \rightarrow v^{\prime}$ transitions (see Figure lb) involve the excitation of the $C-C$ and $C \equiv N$ stretch mode. respectively', we can use the molar absorptivities and $\Gamma$ values shown in Table 1 to obtain a value of $1.7 \times 10^{-12} \mathrm{~cm}^{3} / \mathrm{erg}$. This value is substantially larger than the DOVE-IR values for this same set of vibrational modes because the SPE process is triply resonant.

\section{Six Wave Mixing Methods}

Doubly vibrationally enhanced spectroscopy is also being pursued using six wave mixing (SWM) spectroscopy or coherent 2D Raman spectroscopy ${ }^{44.55}$ Figure $1 \mathrm{c}$ shows the relevant diagrams for an example of these methods. The example shown is the direct analogue to DOVE-IR FWM but instead of infrared transitions. the method uses Raman transitions. As shown. two of the three Raman transitions involve fundamentals where $\Delta v= \pm 1$ and the third involves a combination band. At least one of the transitions must be a combination band in order to have a cross-peak in the two dimensional spectrum.

The $\chi^{(3)}$ expression for the pathway $g g \rightarrow e^{\prime} g \rightarrow v^{\prime \prime} g \rightarrow$ $v^{\prime \prime} e \rightarrow v^{\prime \prime} v^{\prime} \rightarrow e^{\prime \prime} v^{\prime}$ is shown below.

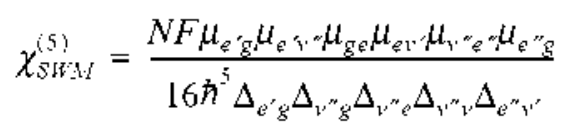

The value of $\chi^{3 i}$ can be estimated using the same approach described for the DOVE-IR and SPE methods if one uses the Raman $\chi^{(3)}$ for the individual vibrational resonances in the SWM process. If we use the $\mathrm{C} \equiv \mathrm{N}$ and $\mathrm{C}-\mathrm{C}$. stretch vibrational modes of acetonitrile. we find the $\chi^{(3)}$ is small because the Raman combination band between these modes is too weak to be observed in the Raman spectrum. We can. however. estimate a value if we use the $\mathrm{C}-\mathrm{H}$ wag $\left(\mathrm{V}_{3}\right)$ and the $\mathrm{C}-\mathrm{C}\left(\mathrm{v}_{4}\right)$ stretch modes because there is a Raman combination band for $v_{3}+v_{4}$. Using the relative intensities of the different Raman lines to estimate the Raman susceptibilities of these modes (see Table 1) and equation 10 . one can estimate the value $5 \times 10^{-39} \mathrm{~cm}^{6} / \mathrm{erg}$ for $x^{(5)}$ corresponding to the transitions where $v^{\prime}$ is the $v_{3}$ mode and $v^{\prime \prime}$ is the $v_{3}+v_{4}$ combination band. The significance of this value must be assessed by comparison with the interfering cascading processes.

The SWM signal is plagued by contributions from cascaded FWM signals ${ }^{5(x)<1}$ Here a four wave mixing process involving three lasers creates an output which together with the other two lasers excites a second four wave mixing process that gives a signal at the same frequency and directionality as the SWM signal. These interfering signals have the same intensity dependence on the laser intensities and overall phase matching conditions so they are difficult to distinguish. They do have different dependences on the sample concentration. pathlength. and the phase matching for the intermediate photon. There are two types of cascaded FWM processes - a sequential cascade where the first FWM process is completed and its output drives the second FWM process and a parallel cascade where the output of the first process occurs after the 
second FWM process has begun so it drives a later part of the second FWM process. ${ }^{58.6 \%}$ An example of the sequential cascade is shown in Figure 1c. One can estimate the relative size of the two contributions by calculating the intensity of the signals from each process. A rough estimate for the ratio of the intensities for the sequential cascade is given by

$$
\frac{I_{\text {cascade }}}{I_{S W H}}=\frac{4 \pi^{2} N^{2} z^{2} M R}{c^{2} n^{2}}
$$

where $R$ is the ratio of the Raman cross-sections and detuning factors for the three different transitions in the cascaded and SWM process and should be near one. Using the parameters shown in Table 1, we calculate the ratio is 3600 for a $1 \mathrm{~mm}$ pathlength. It is therefore necessary to achieve this level of discrimination between the two signals if one is to observe the true SWM process. The largest discrimination is obtained by arranging the phase matching conditions so the output of the first FWM process is not phase mismatched for all of the cascades but the overall phase matching is still maintained ${ }^{57.58 .61 .68}$ Heterodyne detection also provides discrimination because the phase of the output signal differs between the two processes and it also boosts the signal levels. ${ }^{62.63}$ The excitation beams' polarizations can be chosen to optimize the discrimination. ${ }^{64}$ Additional discrimination is possible by using electronic resonances to enhance the signals. ${ }^{56}$ Electronic resonances allow one to work at lower concentrations and shorter pathlengths. As can also be seen from equation 14, the discrimination improves inversely as the square of concentration and path-length. ${ }^{56}$

\section{Conclusions}

The promise of CMDVS methods lies in their ability to provide increased resolution from the multiple dimensions. selectivity for specific components. conformers. isotopomers. etc. structural detail from the $x^{(3)}$ tensor. picosecond temporal resolution as defined by the dephasing rates. and sensitivity to the inter- and intra-molecular interactions that couple modes. ' CMDVS methods can be performed in the frequency or time domain. ${ }^{.}$CMDVS methods that are based upon odd wave mixing processes such as three wave and five wave mixing are inherently surface selective because the molecules with opposite directions interfere in isotropic materials and eliminate their contributions. ${ }^{65-67}$ An $n+1$ wave mixing process involves $n$ excitation pulses and is analogous to an npulse NMR experiment. The relative importance of the higher order resonant processes scales as the ratio of the Rabi frequency to the dephasing rate. $\Omega / \Gamma^{56}$ For high laser intensities that approach saturation. these higher order processes can become quite important. However. these higher order processes will always need to be concerned about cascaded interference so it will be important to solve the cascading problem. When that discrimination is achieved. these higher order processes will offer greater selectivity through their higher dimensionality.
Acknowledgments. This work was supported by the National Science Foundation under grant C.HE-0130947.

\section{References}

1. Wright. J. C. Int. Rev: Phws. Chem. 2002. 21, 185-255.

2. Cho, M. In Adrances in Ifult-Photon Processes and Spectroscopy. Ist ed.: Lin. S. H. Villaeys. A. A.. Fujimura. Y.. Eds.: World Scientific: Singapore. 1999: Vol. 12. pp 1-72.

3. Zhao. W. Wright. J. C. J. An. Chem. Soc 1999. 121. 10994-10998.

4. Mukamel. S. Principles of Nontinear Optical Spectroscopy, lst ed: Oxford University Press: New York, 1995.

5. Shen. Y. R. The Principles of Fonlhear Optics, Wiley Interscience: New York. 1984.

6. Levenson. M. D.: Kan1o. S. S. Introduction to Nonlinear Spectroscopv: Academic Press: New York. 1988.

7. Lee, D. Albrecht, A. C Adhances in Infrored and Rantan Spectroscopy, 1st ed.: Wiley-Heyden: Chichester. 1985; Vol. 12.

8. Richmond. G. L.; Robinson. J. M: Shannon, V. L. Prog. Surf. Sci. 1988. 28. 1 .

9. Wright. J. C. In Applications of Lasers to Chenical Problems. 1st ed.: Evar1s. T. R.. Ed.: John Wiley\&Sons: New York. 1982: p 35.

10. Wright, J. C.: Carlson, R. J.: Hurst. G. B.; Steehler. J. K.: Riebe, M. T.: Price, B. B.; Nguyen. D. C.; Lee. S. H. Int Rev Phws. Chem. 1991, 10, 349-390.

11. Wright. J. C.: Carlson. R. J.: Riebe. M. T.: Steehler. T. K.: Nguyen. D. C.: Lee. S. H.: Price. B. B.: Hurst. G. B. In Iibrational Spectroscopy and Structure. 1st ed.: Bist. H. D.. Durig. J. R.. Sullivan. J. F., Eds.; Elsevier Science Publishers: Amsterdam. 1989: Vol. 17B, pp 123-158.

12. Carlson, R. J.: Wright. J. C. J. Chem. Phus. 1990, 93, 2205-2216.

13. Carlson. R. T.: Wright. T. C. J. Chem Phus. 1990. 92.5186-5195.

14. Carlsont. R. T.: Nguyen. D. C.: Wright. İ. C. J. Chem. Phus. 1990. 92. 1538-1546.

15. Carlson, R. J.: Wright. J. C. Anal Chent 1991, 63. 1449-1451.

16. Chen. P. C.: Hamilton. J. P. Zilian. A.: LaBuda. M. J.: Wright, J. C. Appl. Spectrosc. 1998. 52,380-392.

17. Wright. J. C.: Chen1. P. C.: Hamilton1. J. P.: Zilian1. A.: LaBuda. M. J. Appl. Spectrosc. 1997. $51.949-958$.

18. Hamilton. J. P.: LaBuda. M. T.: Wright. J. C. Chent. Phns. Letters 1997. $277,175-182$

19. LaBuda. M. J; Wright, J. C. Chem. Phns Letters 1998. 290, 29 35 .

20. LaBuda. M. T.: Wright. T. C. J. Chem. Phss. 1998. 108. 4112 4122 .

21. Zhao. W.: Wright. J. C. Phys. Rev: Letters 2000. 84. 1411-1414.

22. Zhao. W. Wright J. C. Phys. Rev Letters 1999. 83 , 1950-1953.

23. Besemann, D. M. Condon. N. J.: Murdoch, K. M.: Mever, K. A.; Zhao. W: Wright, J. C. Chem. Plys. 2001. 266. 177.

24. Murdoch. K. M.: Condont. N. J.: Zhao. W.: Besemant1. D. M.: Meyer. K. A.: Wright. T. C. Chem. Phus. Letters 2001. 335.349.

25. Levensont. M. D.: Bloembergen. N. J. Chent Phws 1974. 60. $1323-1327$.

26. Levenson. M. D.: Bloembergen, N. Phws Ren: $B$ 1974. 10. $4147-$ 4463.

27. Wright. J. C.: Condon. N. I.: Murdoch. K. M.: Besemann. D.: Meyer. K. A. I. Plys. Chem to be published.

28. Kwak. K.: Cha. S.: Cho. M.: Wright. I. C. J. Chent. Phts 2002. 117. $5675-5687$

29. Hamm. P.: Lim, M.: Hochstrasser. R. M. J. Phus Chem. B 1998. 102. $6123-6138$

30. Hamm. P.: Lim. M.: Hochstrasser. R. M. Phus. Rev Letters 1998. $81.5326-5329$.

31. Hamm. P: Lim. M.: DeGrado. W. F.: Hochstrasser. R. M. Proc Natl. Acad Sci 1999. 96, 2036-2041.

32. Hamm. P; Lim. M. DeGrado, W. F.; Hochstrasser, R. M. J. Ph.s. Chem. A 1999. 103. 10049-10053. 
33. Hamm, P.: Lim. M.: DeGrado, W. F: Hochstrasser, R. M. J. Chem. Plys. 2000. 112. 1907-1916.

34. Asplund. M. C.: Zamini. M. T.: Hochstrasser. R. M. Proc. Natl. Acad Sci. 2000. 97. 8219-8224.

35. Asplund. M. C.: Lim. M.: Hochstrasser. R. M. Chem. Phis. Letters 2000. 323. 269-277.

36. Zami, M. T. Asplund. M. C.: Hochstrasser. R. M. J. Chent. Phns. $2001,114,4579-4590$.

37. Zanni. M. T.: Cnanakaran. S.: Stenger. J.: Hochstrasser. R. M. $J$. Plys. Chem. B 2001. ASAP. Ah 8. 2001.

38. Lim. M.: Hamm. P.: Hochstrasser. R. M. Proc. Natl Acad. Sci. $1998,95,15215-15320$.

39. Golonzka, O.: Khalil M.: Denirdoven. N.: Tokmakoti, A. Phus. Rev Letters 2001. 86. 2154-2157.

40. Tokmakoff. A. J. Phs. Chem . 4 2000. 104. $4247-4255$.

41. Khalil. M.: Tokmakotf. A. Chem. Phys. 2001. 266. 213-230.

42. Khalil. M.: Demirdoven. N.: Golonzka. O.: Fecko. C. J.: Tokmakott. A. J. Phis. Chem A 2000, 104.5711-5715.

43. Khalil. M.: Golonzka, O. Demirdowen. N.: Fecko, C. J.: Tokmakoft. A. Chem. Phys. Letters 2000, 321, 231-237.

44. Tominaga. K.: Yoshihara. K. Phis. Rev .4 1997. 55.831.

45. Tominaga. K.: Keogh. G. P.: Naitoh. Y: Yoshihara. K. J. Ramam Spect: 1995, 26. 495-501.

46. Tominaga, K.: Yoshihara, K. Phns. Rev Letters 1995, 74, 30613064 .

47. Tominaga, K.: Yoshihara. K. J. Chem. Phns. 1996. 104. 44194426 .

48. Tokmakoti. A.: Fleming. G. R. J. Chem. Phns. 1997. 106. 25692582.

49. Tokmakoff. A.: Lang M. I.: Larsen, D. S.: Fleming, G. R. Chent. Plys Letters 1997, 272, 48-54.
50. Tokmakoff, A.: Lang, M. J.: Larsen, D. S.: Fleming. G. R.: Chemvak. V: Mukamel, S. Phns. Res: Letters 1997. $79.2702-2705$.

51. Steffetr. T.: Fourkas. T. T.: Duppent. K. J. Chem. Phus. 1996. 105. $7364-7382$

52. Steffen. T.: Duppen1. K. J. Chem. Phws. 1997. 106.3854-3864

53. Steffen. T.; Duppen. K. Chem. Phys. Letters 1997, 273,47-54

54. Steffen. T.: Duppen. K. Phys. Rev: Letters 1996. 76, 1224-1227.

55. Duppen. K.: Molenkamp, L. W.: Morsink. J. B. W.: Wiersma, D. A.: Trommsdortf. H. P. Chem. Phus, Letters 1981. 84. 421-424.

56. Ivanecky. T. E.: Wright. T. C. Chem. Phus. Letters 1993. 206. 437. 444

57. Blank. D. A.: Kaufman, L. J.; Fleming. G. R. J. Chent. Phns 2000. 113.771-778

58. Blank. D. A.: Kautman, L. J; Fleming. G. R. J. Chem. Plys. 1999.111.3105-3114.

59. Kirkwood. J. C.: Unness. D. T.: Albrecht. A. C.: Stimson. M. T. Chent Phys. Letters 1998. 293.417-422.

60. Ulness. D. J.: Kirkwood, J. C.: Albrecht, A. C. J. Chent. Phws 1998. $108,3897-39002$

61. Astinov. V.: Kubarych, K. J:- Milne, C. J:- Miller. R. J. D. Chem Phus. Letters 2000. 327. 334-342.

62. Kubarych. K. J.: Milne. C. J.: Lin1. S.: Astinov. V: Miller. R. T. D. J. Chem. Phs 2002. 116. 2016-2042.

63. Kaufman. L. J.: Heo. J; Ziegler. L. D.: Fleming. G. R. Phys. Rer: Letters 2002, $88,2074012-207406$.

64. Kaufman. L. J.: Blank, D. A.; Fleming. G. R. J. Chem. Phys 2001. $11+.2312-2332$.

65. Richmond. G. L. Anal Chem 1997.69.537A

66. Richmond. G. L. Amm. Ren: Phws Chem. 2001. 52. 257-265.

67. Com. R. M.: Higgins. D. A. Chem. Rev 1994. 94, 107-125. 